\title{
Brain dopamine-serotonin vesicular transport disease
}

INSERM

\section{Source}

INSERM. (1999). Orphanet: an online rare disease and orphan drug data base. $\underline{\text { Brain }}$ dopamine-serotonin vesicular transport disease. ORPHA:352649

Brain dopamine-serotonin vesicular transport disease is a newly discovered infantileonset neurometabolic disease characterized by dystonia, parkinsonism, nonambulation, autonomic dysfunction, developmental delay and mood disturbances. 